\title{
Weird spikes
}

\author{
Chao-Yu Chen ${ }^{1}$ and Ju-Yi Chen ${ }^{2}$ \\ ${ }^{1}$ National Cheng Kung University Hospital \\ ${ }^{2}$ Institute of Clinical Medicine, College of Medicine, National Cheng Kung University, \\ Tainan, Taiwan
}

June 18, 2020

A 74-year-old man with a history of severe mitral stenosis had received bioprosthetic mitral valve replacement. Ten years later, an epicardial pacemaker with VVIR mode was implanted because of sick sinus syndrome. Three years later, symptomatic junctional bradycardia (Panel A) and epicardial lead fracture were suspected, based on high lead impedance. A dual-chamber rate-modulated (DDDR) pacemaker was implanted through the left subclavian vein, and the epicardial pacemaker was left in place (Panel B, Red circle), with shift to OVO mode. The 12-lead electrocardiogram revealed atrial and ventricular dual-paced rhythm (Panel C). Five years after implantation of the DDDR-mode permanent pacemaker, pacing-induced cardiomyopathy, with left ventricle (LV) ejection fraction of $34 \%$ even with optimized medical therapy, was noted. Cardiac resynchronization therapy (CRTP) was upgraded (Panel D). Although the biventricular pacing functioned well initially (Panel E), high LV pacing threshold (Panel F) prompted us to adjust the LV lead from bipolar to unipolar (LV lead to can) pacing. The patient became less symptomatic after receiving CRTP implantation. However, weird pacing spikes, with regular $65 \mathrm{bpm}$ without interference of the biventricular pacing, were present. These spikes were unaffected by adjusting the CRTP mode from DDD to VVI (Panel F). We subsequently learned that the pacing spike was generated by the unremoved epicardial pacemaker. The pacemaker reached elective replacement indication and started to pace at VVI mode of $65 \mathrm{bpm}$. Because of sensing and capture failure, the epicardial pacemaker had no influence on the CRTP, but it continued to produce spikes on the electrocardiogram. 

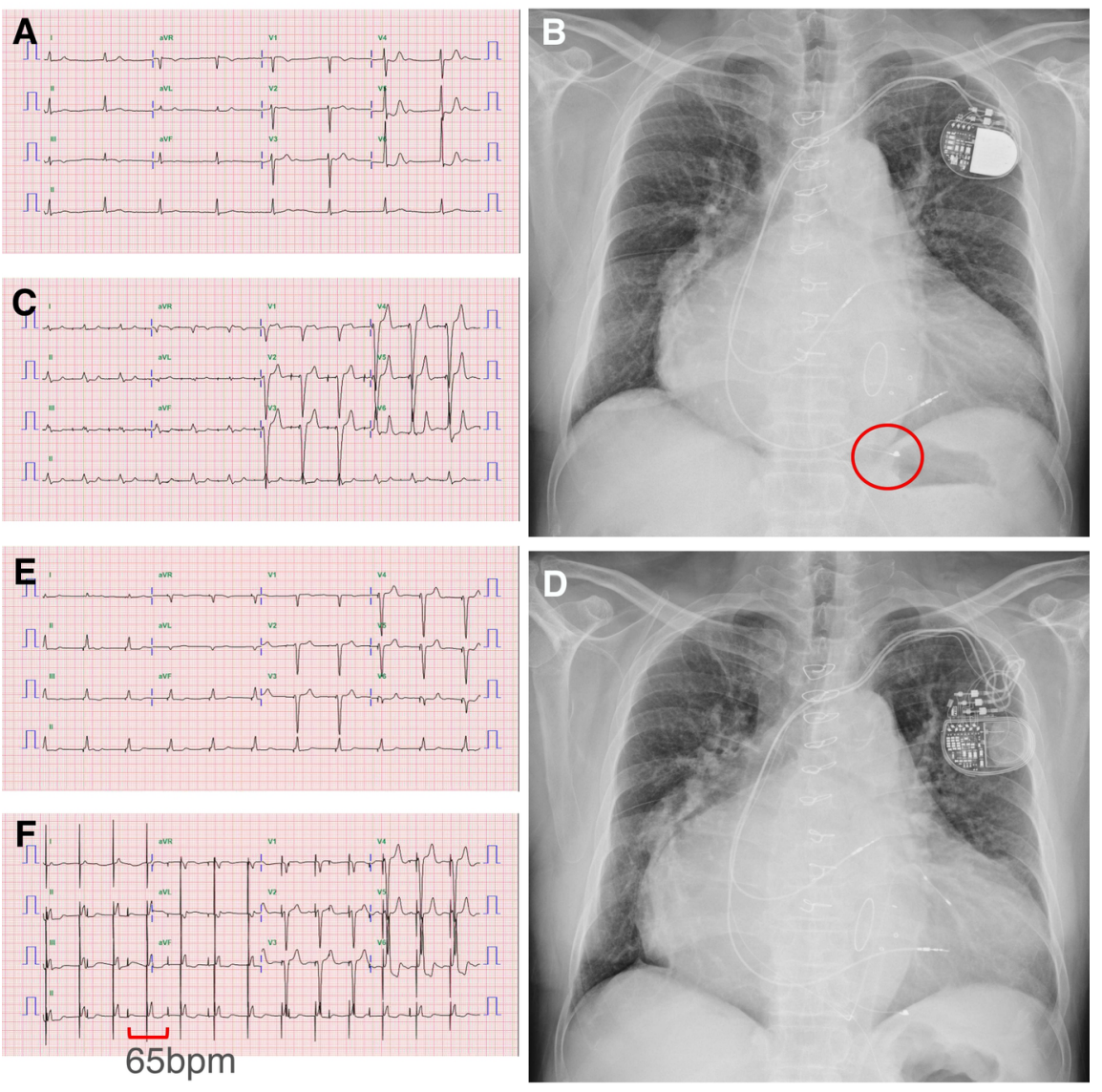